Accepted

Biometrical Journal 45 (2003) 7, 1-19

\title{
Parameter Redundancy in Multistate Capture-Recapture Models
}

\author{
Olivier Gimenez* ${ }^{*}$, Rémi Choquet and Jean-Dominique Lebreton \\ Equipe Biométrie et Biologie des Populations, Centre d'Ecologie Fonctionnelle et Evolutive CNRS, \\ 1919 route de Mende, 34293 Montpellier, FRANCE
}

\section{Abstract}

Multistate capture-recapture models are a powerful tool to address a variety of biological questions concerning dispersal and/or individual variability in wild animal populations. However, biologically meaningful models are often over-parameterized and consequently some parameters cannot be estimated separately. Identifying which quantities are separately estimable is crucial for proper model selection based upon likelihood tests or information criteria and for the interpretation of the estimates obtained. We show how to investigate parameter redundancy in multistate capture-recapture models, based on formal methods initially proposed by Catchpole and his associates for exponential family distributions (Catchpole, Freeman and Morgan, 1996. Journal of the Royal Statistical Society Series B 58, 763-774). We apply their approach to three models of increasing complexity.

Key words: Capture-Recapture; Multistate Models; Parameter redundancy; ArnasonSchwarz model; Heterogeneity of Capture; Combination of Information.

\section{Introduction}

Multistate capture-recapture models (ARNASON, 1973; SCHWARZ et al., 1993; LebRETON and PRADEL, 2002) are a natural generalization of the single state CormackJolly-Seber (CJS) model (CoRMACK, 1964; Jolly, 1965; SEbER, 1965) for the estimation of survival probabilities (and possibly population size) using capture (or resightings) of marked individuals (LEBRETON et al., 1999). In multistate models, individuals can die, or survive and be captured as in single state models, but they can also move between states, according to probabilities of transition between states. States can be either geographical sites (multisite models) or states of categorical variables defined at the individual level, such as, e.g., reproductive state. As a consequence, a great flexibility is reached in modeling biological phenomena (Nichols and Kendall, 1995; Lebreton and Pradel, 2002) and in addressing a variety of biologically relevant questions. These questions concern for instance:

- Dispersal (states = geographical sites, Hestbeck et al., 1991)

- Trade-off between reproductive status and survival (states = reproducer/nonreproducer, Nichols et al., 1994)

* Corresponding author: olivier.gimenez@cefe.cnrs.fr 
- Rate of accession to reproduction (states = reproducer/non-reproducer, PRADEL and LEBRETON, 1999; states = reproducer/non-reproducer together with age-dependence, LEBRETON et al., 2003).

Moreover, both special cases of the basic time-dependent models obtained by constraining parameters (BROWNIE et al., 1993), and generalizations, such as models with a memory of previous states occupied (BROwNIE et al., 1993) have been proposed. Lebreton et al. (2003) and Lebreton and Pradel (2002) describe further generalizations, in particular age-dependent multistate models to estimate recruitment and age-dependent dispersal. Implementation of these generalizations has been made possible largely by the development of appropriate (White and BuRnhAM, 1999; CHOQUET et al., 2003) and have greatly increased the biological interest of multistate models, which appear as a natural tool for modeling complex individual capture-histories (LEBRETON and PRADEL, 2002).

However, because the scalar parameters of single state models are replaced by matrices of transition probabilities between states and the scalar recapture probabilities by vectors of state-dependent recapture probabilities, multistate models tend in general to have a large number of basic parameters. Moreover, the probabilities of capture histories are more involved than in the single state case, with sums of products of probabilities rather than just products. Such a likelihood tends to behave like mixture models and may produce multiple maxima (LEBRETON and PRADEL, 2002). As another important consequence, multistate capture-recapture models commonly present parameter redundancy problems (LEBRETON and PRADEL, 2002). A model is said here to be parameter redundant, if its likelihood can be expressed as a function of fewer functions of the parameters than the original number of parameters (CATCHPOLE et al., 1996). This number of functions of the parameters, of which unique maximum likelihood estimates can be obtained, will be called for short the number of estimable parameters. Determining how many and which functions of the original parameters are estimable is crucial in the interpretation of estimates and in model selection (VIALLEFONT et al., 1998; BURNHAM and ANDERSON, 1998).

Parameter redundancy is also present in models belonging to the CJS family (LEBRETON et al., 1992), such as models with immediate trap dependence (PRADEL, 1993), or the standard time-dependent CJS model sensu stricto, in which the last survival probability and the last recapture probability cannot be estimated separately, only their product being estimable. As mentioned above, parameter redundancy problems tend to be worse for multistate than for single state models, in particular when age-dependence, that further increases the number of parameters, is considered. Constraints on parameters, in the spirit of generalized linear models (McCullagh and Nelder, 1989) as commonly used for CJS models (LeBRETON et al., 1992), make it possible to reach greater parsimony, i.e. fewer parameters, and, at the same time to focus on biological relevance. However, reducing the number of parameters starting from a model with complex redundancy problems does not guarantee the absence of parameter redundancy in the final model.

2031633 Biom. Journal (7/2003) Art.: 0580/Gimenez 3B2 Bearb.: Sch.

SIGNA 18.7.2003 ges.Seiten $19 \quad\{$ _1 19 bio/bio03_07/0580/bio0580u.3d

Literaturstellen: 41 <bibitem> nur für Literatur verwenden, keine Leerzeilen damit formatieren, die exakte Anzahl der LitStellen wird für SGML benötigt 
Clearly, a general approach is needed to address parameter redundancy problems in multistate models (LEBRETON and PRADEL, 2002).

VIALLEFONT et al. (1998) proposed for single-state capture recapture models a numerical approach based on the rank of the matrix of the second order derivatives of the log-likelihood with respect to the parameters. A formal approach to determine the number of estimable parameters has been proposed for the wide class of exponential model family by Catchpole, Morgan and Freeman (CMF in what follows) in a series of papers (1996, 1997, 1998 and 2001). The CMF approach is based on the symbolic calculation of the rank of a matrix of first order derivatives of probabilities with respect to the parameters.

The purpose of this paper is to develop and provide general tools in order to apply the CMF approach to multistate capture-recapture models. We first recall general results about parameter redundancy and the CMF approach, and complete them by some specific results (Section 2). Then we present general results on time-dependent multistate capture-recapture models in relation with the CMF approach (Section 3). We develop three examples of increasing complexity:

- The Arnason-Schwarz model (ARNASOn, 1973; SchwARZ et al., 1993), which is a straightforward generalization of the time-dependent CJS model. Its generality makes a good knowledge of estimable parameters crucial.

- A multistate model for mixtures of live recaptures and of dead recoveries (LEBRETON et al., 1999).

- A multistate model presentation of a survival model with immediate trap dependence (PRADEL, 1993).

The last two models can easily be generalized, and our purpose is to provide general tools that can be used when building any particular model. To this effect, following again the rationale in the CMF approach, we developed symbolic computation code using software Maple. Besides the calculation of the number of estimable parameters, which adapts the existing CMF approach, the symbolic calculation of estimable functions of parameters is new. It could obviously be used with success for single state capture-recapture models. The commented code for our three examples is available at URL: ftp://ftp.cefe.cnrs-mop.fr/biom/PRM.

\section{The Catchpole, Morgan and Freeman (CMF) approach}

Multistate models belong to the wide class of product-multinomial distributions depending on a vector of parameters $\theta \in[0,1]^{p}$. We denote as $\mu_{i}(\theta)$ the vector of all multinomial probabilities, with the last probability of each multinomial skipped to avoid unnecessary calculation (Catchpole and Morgan, 1997), and as $n$ its dimension. The following developments can easily be extended to any distributions belonging to the exponential family. A model is said to be parameter redundant if $\mu=\left(\mu_{1}, \ldots, \mu_{n}\right)^{T}$ can be expressed in terms of $q$, but not less than $q$, functions $\beta=\left(\beta_{1}, \ldots, \beta_{q}\right)^{T}$ called a minimal parameter set where $q<p$. Clearly any one- 
to-one transformation of a minimal parameter set is another minimal parameter set. The CMF approach consists of the following steps:

- Step 1: Form the vector of log-probabilities $\log (\mu(\theta))$. The logarithm is used here for convenience (CATCHPOLE and Morgan, 1997).

- Step 2: Differentiate with respect to $\theta$, i.e., obtain the $n \times p$ matrix $A(\theta)$ with generic term $A(\theta)_{l, i}=\frac{\partial \log \left(\mu_{i}\right)}{\partial \theta_{l}}$.

- Step 3: Determine the rank of $A(\theta)$. The key result of the CMF approach is that rank $(A(\theta))$, equals $q(q \leq p)$, the dimension of $\beta$. Then, if the model is not full rank, i.e., is parameter redundant:

- Step 4: Determine the $d=p-q$ linearly independent solutions $\alpha_{j}(\theta)=\left(\alpha_{1, j}(\theta), \ldots, \alpha_{l, j}(\theta), \ldots, \alpha_{p, j}(\theta)\right)^{T}(j=1, \ldots, d)$ of the equation

$$
\alpha(\theta)^{T} A(\theta)=0
$$

where $\alpha(\theta)$ forms a base of the left kernel (GoluB and VAN LOAN, 1996) of $A(\theta)$.

- Step 5: Inspect the $\alpha s$. The positions of the $s$ common zero entries correspond to the components $\theta_{l}$ of $\theta$ which are separately estimable, i.e. the indices $l$ for which $\alpha_{l}, j(\theta)=0$ for all $j$. It remains to exhibit the $q-s$ complex estimable functions of the $c=p-s$ parameters corresponding to nonzero entries in $\alpha$.

- Step 6: These functions $f$ are obtained by solving the following system of $d$ linear first-order partial differential equations (PDEs)

$$
\sum_{l=1}^{p} \alpha_{l, j} \frac{\partial f}{\partial \theta_{l}}=0, j=1, \ldots, d,
$$

We propose to use symbolic calculus to solve such equations (CHEB-TERrAB and VON BulOw, 1995), and will develop appropriate tools using software Maple.

A minimal parameter set $\beta$ (CATChPOLE and Morgan, 1998) is made up of the $s$ parameters that are separately estimable and of the $q-s$ functions determined in step 6. For instance for the CJS model with $k$ capture occasions (e.g. LEBRETON et al., 1992), there are $p=2 k-2$ parameters, but only $q=2 k-3$ are separately estimable, $\phi_{1}, \phi_{2}, \ldots, \phi_{k-2}, p_{2}, p_{3}, \ldots, p_{k-1}(s=2 k-4)$ and the product $\phi_{k-1} p_{k}$ $(q-s=1)$. The rank deficiency is equal to 1 (for a detailed treatment of the CJS model, see CATCHPOLE et al., 2002).

The calculation of rank $(A(\theta))$ in step 3 can be made simpler by considering the decomposition $L(\theta)=D(\theta) A(\theta) B(\theta)$. If $D(\theta)$ and $B(\theta)$ are square matrices of full rank, i.e., non-singular matrices, then $\operatorname{rank}(L(\theta))=\operatorname{rank}(A(\theta))$ (GRAYBILL, 1969 , th. 1.6.7 p.13). $L(\theta)$ and $A(\theta)$ are then said to be equivalent matrices (GRAYBILL, 1969, p. 11). We recommend applying successive pre- and postmultiplication by matrices $D(\theta)$ and $B(\theta)$ that remain full rank for any $\theta$ to make simple patterns appear in $L(\theta)$. This novel approach is particularly useful for distinguishing between essentially $(\forall \theta, \operatorname{rank}(A(\theta))=q)$ or conditionally 
$\left(\exists \theta^{*}\right.$, rank $\left(A\left(\theta^{*}\right)\right)=q$ ) full rank models (CATChPOle and Morgan, 1997). A model is essentially full rank when $\forall \theta$, rank $(L(\theta))=q$ and conditionally full rank otherwise. In some cases, $L(\theta)$ can be made of constants which implies in a straightforward way that its rank does not vary with $\theta$. In the appendix, we provide the reader with an example.

In the CMF approach, the rank is obtained for a model at a given size, i.e., for particular values of $n$ and $p$. CATCHPOLE and MoRgAn $(1997,2001)$ use extension theorems to generalize a rank calculation for a model under a particular number of capture occasions to the same model under an arbitrary number of capture occasions. In the appendix, we develop the extension theorem needed for multistate capture-recapture models.

\section{Multistate models and the CMF approach}

In time-dependent multistate CMR models, individuals move among successive occasions ( $k$ possible occasions) between $a$ discrete states (for 2 or more states). The following standard notation (BROwNIE et al., 1993) is used:

$\Phi_{i}$ is the $a \times a$ matrix of the survival/movement probabilities $\phi_{i}^{r, s}$ between times $i$ and $i+1(1 \leq i \leq k-1)$ and from state $r$ to $s$. To make further calculations simpler, the row and the column indices are the departure state and the arrival state, respectively.

$P_{j}$ is the $a \times 1$ vector of the probability of capture $p_{j}^{r}$ in state $r$ at occasion $j$ $(2 \leq j \leq k)$.

The time-dependent multistate CMR model described above has been proposed by Arnason (1973, see also Schwarz et al., 1993), it relies on the usual CMR model assumption of independence and homogeneity of individuals (LEBRETON et al. 1992). For a given occasion of marking, the number of individuals in the various possible recapture histories follow multinomial distributions. The multistate $m$-array (BROwNIE et al., 1993) is a set of sufficient statistics (Table 1) that cannot be reduced any further under this specific model.

Let $m_{i}^{r}$ be the vector of the numbers next recaptured over occasions $j=i+1, \ldots, k$ in states $r=1, \ldots, a$, i.e. the row in the multistate $m$-array corresponding to releases in state $r$ at occasion $i$. Let $r_{i}^{r}$ be the number never recaptured. Conditional on the number of individuals released at occasion $i$ in state $r$, say $R_{i}^{r},\left(m_{i}^{r}, r_{i}^{r}\right)$ follows also a multinomial distribution. This well-known property of time-dependent models results from the assumed independence of future and past history for each individual. In particular $R_{i}^{r}$ is made of newly marked and already marked individuals. Accounting for redundancy in multinomial probabilities, the probability corresponding to $r_{i}^{r}$ can be dropped. As a consequence, the probabilities that an individual last captured in state $r$ at occasion $i$ is recaptured in state $s$ at occasion $j, \Omega_{i, j}^{r, s}$ can thus be used as $\mu_{i}(\theta)$ for applying the CMF approach (CATCHPOLE and MORGAN, 1997). 
Table 1

The multistate $m$-array (BROWNIE et al., 1993): a synthetic representation of the number of individuals released in state $r$ in year $i$ and recaptured in state $s$ in year $j$ ( $a$ states and $k$ years)

\begin{tabular}{|c|c|c|c|c|c|c|}
\hline $\begin{array}{l}\text { year } \\
\text { of release }\end{array}$ & $\begin{array}{l}\text { number } \\
\text { released }\end{array}$ & $\begin{array}{l}\text { year } \\
2\end{array}$ & $\begin{array}{l}\text { of } \\
3\end{array}$ & $\begin{array}{l}\text { recapture } \\
\ldots\end{array}$ & $k$ & $\begin{array}{l}\text { number } \\
\text { never } \\
\text { recaptured }\end{array}$ \\
\hline
\end{tabular}

\begin{tabular}{lllllll}
\hline 1 & $R_{1}$ & $m_{1,2}$ & $m_{1,3}$ & $\ldots$ & $m_{1, k}$ & $r_{1}$ \\
2 & $R_{2}$ & & $m_{1,2}$ & $\ldots$ & $m_{2, k}$ & $r_{2}$ \\
$\vdots$ & $\vdots$ & & & $\ddots$ & $\vdots$ & $\vdots$ \\
$k-1$ & $R_{k-1}$ & & & & $m_{k-1, k}$ & $r_{k-1}$
\end{tabular}

$R_{i}(i=1, \ldots, k-1) \quad$ are $a \times 1 \quad$ vectors $\quad$ with elements $\quad R_{i}^{r} ; \quad m_{i, j}(i=1, \ldots k-1$; $j=i+1, \ldots, k)$ are $a \times a$ matrices with elements $m_{i, j}^{r, s} ; r_{i}(i=1, \ldots, k-1)$ are $a \times 1$ vectors with elements $r_{i}^{r}$.

In block matrix notation, the matrix of these probabilities, arranged as in the multistate $m$-array is (Brownie et al., 1993, Table 2):

$$
\Omega=\left[\begin{array}{cccc}
\Phi_{1} D\left(P_{2}\right) & \Phi_{1} D\left(1-P_{2}\right) \Phi_{2} D\left(P_{3}\right) & \ldots & \Phi_{1} D\left(1-P_{2}\right) \ldots \Phi_{k-1} D\left(P_{k}\right) \\
0 & \Phi_{2} D\left(P_{3}\right) & \ldots & \vdots \\
\vdots & \vdots & \ddots & \vdots \\
0 & 0 & \ldots & \Phi_{k-1} D\left(P_{k}\right)
\end{array}\right]
$$

where $D$ is the diagonal operator and 1 is a $(a \times 1)$ vector of ones.

The CMF approach proceeds then as follows:

- Step 1: Arrange the nonzero elements of $\Omega$ in a vector $\mu$ of length $a^{2} \frac{k(k-1)}{2}$ obtained in Maple by stacking the columns of the lower triangular part of $\Omega^{T}$.

- Step 2: Obtain the derivative matrix $A$ by formal differentiation:

$$
A_{l, i}=\frac{\partial \log \mu_{i}}{\partial \theta_{l}}, \quad 1 \leq l \leq p, \quad 1 \leq i \leq a^{2} \frac{k(k-1)}{2}
$$

- Following steps 3-6, depending on the model under study, as examined in the examples.

\section{Example 1: the Arnason-Schwarz model}

This model, described above (see Section 3), was introduced by ARNASON (1973) and SCHWARZ et al. (1993). Let's assume there are $a=2$ states and $k=3$ capture 
occasions. Then (3) becomes:

$\Omega=\left[\begin{array}{cccc}\phi_{1}^{11} p_{2}^{1} & \phi_{1}^{12} p_{2}^{2} & \left(\phi_{1}^{11}\left(1-p_{2}^{1}\right) \phi_{2}^{11}+\phi_{1}^{12}\left(1-p_{2}^{2}\right) \phi_{2}^{21}\right) p_{3}^{1} & \left(\phi_{1}^{11}\left(1-p_{2}^{1}\right) \phi_{2}^{12}+\phi_{1}^{12}\left(1-p_{2}^{2}\right) \phi_{2}^{22}\right) p_{3}^{2} \\ \phi_{1}^{21} p_{2}^{1} & \phi_{1}^{22} p_{2}^{2} & \left(\phi_{1}^{21}\left(1-p_{2}^{1}\right) \phi_{2}^{11}+\phi_{1}^{22}\left(1-p_{2}^{2}\right) \phi_{2}^{21}\right) p_{3}^{1} & \left(\phi_{1}^{21}\left(1-p_{2}^{1}\right) \phi_{2}^{12}+\phi_{1}^{22}\left(1-p_{2}^{2}\right) \phi_{2}^{22}\right) p_{3}^{2} \\ 0 & 0 & \phi_{2}^{11} p_{3}^{1} & \phi_{2}^{12} p_{3}^{2} \\ 0 & 0 & \phi_{2}^{21} p_{3}^{1} & \phi_{2}^{22} p_{3}^{2}\end{array}\right]$.

The derivative matrix with respect to $\theta=\left(\phi_{1}^{11}, \phi_{1}^{21}, \phi_{1}^{12}, \phi_{1}^{22}, \phi_{2}^{11}, \phi_{2}^{21}, \phi_{2}^{12}\right.$, $\left.\phi_{2}^{22}, p_{2}^{1}, p_{2}^{2}, p_{3}^{1}, p_{3}^{2}\right)^{T}$ is:

$$
A(\theta)=\left[\begin{array}{ccccc}
\frac{1}{\phi_{1}^{11}} & 0 & \cdots & 0 & 0 \\
0 & 0 & \cdots & 0 & 0 \\
\vdots & \vdots & \ddots & \vdots & \vdots \\
0 & 0 & \cdots & \frac{1}{p_{3}^{1}} & 0 \\
0 & 0 & \cdots & 0 & \frac{1}{p_{3}^{2}}
\end{array}\right]
$$

where only the two first and last terms of rows and columns are given.

The rank of $A$ calculated using Maple (Step 3) is $q=10$. Hence, from the original number of parameters $p=12$, one gets the rank deficiency $d=2$, the size of the solution space of equation (1). Hence, to know which parameters - or functions of the parameters - are estimable, one must solve (1) (Step 4). The 2 independent solutions are:

$$
\left.\begin{array}{l}
\alpha_{1}=\left(0,0,0,0,-\frac{\phi_{2}^{11}}{p_{3}^{1}},-\frac{\phi_{2}^{21}}{p_{3}^{1}}, 0,0,1,0\right)^{T} \\
\alpha_{2}=\left(0,0,0,0,0,0,-\frac{\phi_{2}^{12}}{p_{3}^{2}},-\frac{\phi_{2}^{22}}{p_{3}^{2}}, 0,0,0,1\right)^{T}
\end{array}\right\} .
$$

Step 5, i.e., comparing with $\theta$ the $s=6$ zero entries shows that the elements of the matrices $\Phi_{1}$ and $P_{2}$ are separately estimable. There are then $q-s=4$ functions of the $c=p-s=6$ parameters $\phi_{2}^{11}, \phi_{2}^{21}, \phi_{2}^{12}, \phi_{2}^{22}, p_{3}^{1}, p_{3}^{2}$ that are also estimable. These functions are obtained by solving (step 6) the following system of $d=2$ PDEs:

$$
\left.\begin{array}{c}
\frac{\partial f}{\partial \phi_{2}^{11}} \frac{\phi_{2}^{11}}{p_{3}^{1}}+\frac{\partial f}{\partial \phi_{2}^{21}} \frac{\phi_{2}^{21}}{p_{3}^{1}}-\frac{\partial f}{\partial p_{3}^{1}}=0 \\
\frac{\partial f}{\partial \phi_{2}^{12}} \frac{\phi_{2}^{12}}{p_{3}^{2}}+\frac{\partial f}{\partial \phi_{2}^{22}} \frac{\phi_{2}^{22}}{p_{3}^{2}}-\frac{\partial f}{\partial p_{3}^{2}}=0
\end{array}\right\}
$$


The solutions of (6) given by Maple are the $s=6$ parameters $\phi_{1}^{11}, \phi_{1}^{21}, \phi_{1}^{12}, \phi_{1}^{22}$, $p_{2}^{1}, p_{2}^{2}$ with the $q-s=4$ combinations $\frac{\phi_{2}^{21}}{\phi_{2}^{11}}, \frac{\phi_{2}^{22}}{\phi_{2}^{12}}, \phi_{2}^{11} p_{3}^{1}, \phi_{2}^{12} p_{3}^{2}$, so that the likelihood of the Arnason-Schwarz model can be written down in terms of the elements of the minimal parameter set $\beta=\left(\phi_{1}^{11}, \phi_{1}^{21}, \phi_{1}^{12}, \phi_{1}^{22}, \quad p_{2}^{1}, p_{2}^{2}\right.$, $\left.\frac{\phi_{2}^{21}}{\phi_{2}^{11}}, \frac{\phi_{2}^{22}}{\phi_{2}^{12}}, \phi_{2}^{11} p_{3}^{1}, \phi_{2}^{12} p_{3}^{2}\right)$. The set of solutions that we expected by analogy with the single state case - namely the parameters $\phi_{1}^{11}, \phi_{1}^{21}, \phi_{1}^{12}, \phi_{1}^{22}, p_{2}^{1}, p_{2}^{2}$ with the elements of the last occasion matrix product $\Phi_{2} D\left(P_{3}\right)$ - is also a solution of (6). In other words, there exists a one-to-one transformation between these two minimal parameter sets.

Note that in the $\beta$ re-parameterization, the Arnason-Schwarz model is only conditionally full rank since, e.g., the constraints $\phi_{i}^{11}=\phi_{i}^{21}$ and $\phi_{i}^{12}=\phi_{i}^{22}$ or $\phi_{i}^{11}=\phi_{i}^{21}=\phi_{i}^{12}=\phi_{i}^{22}(j=1,2)$ induce a rank deficiency of 1 .

The results above apply to other parameterizations such as those decomposing $\phi_{i}^{r, s}$ into survival probabilities and movement probabilities as $\phi_{i}^{r, s}=s_{i}^{r} \psi_{i}^{r, s}$ where $s_{i}^{r}$ is a state and purely time dependent survival probability and $\psi_{i}^{r, s}$ is the probability of movement (or transition) from the state $r$ at time $i$ to the state $s$ at $i+1$ conditional on survival (HESTBECK et al., 1991). Accounting for the inherent redundancy in parameters wherein $\psi_{i}^{r, r}$ does not appear and is replaced by $1-\sum_{\substack{s=1 \\ s \neq r}}^{a} \psi_{i}^{r, s}$ the following quantities are separately estimable: $s_{1}^{1}\left(1-\psi_{1}^{12}\right), s_{1}^{2}\left(1-\psi_{1}^{21}\right), s_{1}^{2} \psi_{1}^{21}$, $s_{1}^{1} \psi_{1}^{12}, p_{2}^{1}, p_{2}^{2}$ and $s_{2}^{1}\left(1-\psi_{2}^{12}\right) p_{3}^{1}, s_{2}^{2} \psi_{2}^{21} p_{3}^{1}, s_{2}^{1} \psi_{2}^{12} p_{3}^{2}, s_{2}^{2}\left(1-\psi_{2}^{21}\right) p_{3}^{2}$.

The above is for $a=2$ and $k=3$. In Appendix 1, we show that the deficiency is equal to $a=2$ for the Arnason-Schwarz model whatever the number of occasions of capture.

\section{Example 2: multistate recapture models and mixtures of information}

LEBRETON et al. (1999) show how to represent, as specific multistate models, mixtures of information such as live recapture and dead recoveries (BURNHAM, 1993). Mixing different types of events is expected to improve the precision of survival estimates (LEBRETON et al., 1995) and to permit access to parameters otherwise non-estimable (LEBRETON et al., 1999). Applications of this type of models can be found in FreDERIKSEN and BREGNBALLE (2000) for instance.

We represent a time-dependent mixture model with $a=3$ states: state 1, observable, with probability of capture $p$, is 'Alive'; state 2 , with probability of capture $\lambda$, is 'Newly Dead'; state 3, non-observable, is 'Dead' (for further details, see LEBRETON et al., 1999); let also $S$ be the survival probability. Consequently, the 
matrices of transition and recapture probabilities are as follows:

$$
\begin{aligned}
\Phi_{i} & =\left[\begin{array}{ccc}
S_{i} & 1-S_{i} & 0 \\
0 & 0 & 1 \\
0 & 0 & 1
\end{array}\right], \quad i=1, \ldots, k-1 ; \\
P_{j} & =\left[\begin{array}{c}
p_{j} \\
\lambda_{j} \\
0
\end{array}\right], \quad j=2, \ldots, k .
\end{aligned}
$$

Let $k=3$ and let $\theta=\left(S_{1}, S_{2}, \lambda_{2}, \lambda_{3}, p_{2}, p_{3}\right)^{T}$ be the vector of parameters. Step 1 gives the matrix of probabilities:

$$
\Omega=\left[\begin{array}{cccccc}
S_{1} p_{2} & \left(1-S_{1}\right) \lambda_{2} & 0 & S_{1} \bar{p}_{2} S_{2} p_{3} & S_{1} \bar{p}_{2} \bar{S}_{2} \lambda_{3} & 0 \\
0 & 0 & 0 & 0 & 0 & 0 \\
0 & 0 & 0 & 0 & 0 & 0 \\
0 & 0 & 0 & S_{2} p_{3} & \bar{S}_{2} \lambda_{3} & 0 \\
0 & 0 & 0 & 0 & 0 & 0 \\
0 & 0 & 0 & 0 & 0 & 0
\end{array}\right],
$$

where $\bar{p}_{2}=1-p_{2}$, etc. By considering only nonzero elements and working from left to right along the rows of (7), Step 2 permits to write down the matrix of derivatives:

$$
A(\theta)=\left[\begin{array}{cccccc}
1 / S_{1} & -1 / \bar{S}_{1} & 1 / S_{1} & 1 / S_{1} & 0 & 0 \\
0 & 0 & 1 / S_{2} & -1 / \bar{S}_{2} & 1 / S_{2} & -1 / \bar{S}_{2} \\
0 & 1 / \lambda_{2} & 0 & 0 & 0 & 0 \\
0 & 0 & 0 & 1 / \lambda_{3} & 0 & 1 / \lambda_{3} \\
1 / p_{2} & 0 & -1 / \bar{p}_{2} & -1 / \bar{p}_{2} & 0 & 0 \\
0 & 0 & 1 / p_{3} & 0 & 1 / p_{3} & 0
\end{array}\right] .
$$

Its rank deficiency $d$ is 1 so that the difference with the previous example is that the solution space of (1) is only one-dimensional. Solving (1) formally leads to $\alpha=\left(0, \frac{1-S_{2}}{\lambda_{3}}, 0,1,0, \frac{p_{3}\left(S_{2}-1\right)}{S_{2} \lambda_{3}}\right)$. Hence, parameters $S_{1}, \lambda_{2}$, and $p_{2}$ are separately estimable (Step 3). Since the number of zero entries $s$ is 3, there remains $q-s=2$ estimable combinations of $p-s=3$ parameters, $S_{2}, \lambda_{3}$, and $p_{3} \mathrm{Z}$ (Step 5). These are obtained by solving the single PDE (Step 6):

$$
\frac{\partial f}{\partial S_{2}} \frac{1-S_{2}}{\lambda_{3}}+\frac{\partial f}{\partial \lambda_{3}}+\frac{\partial f}{\partial p_{3}} \frac{p_{3}\left(S_{2}-1\right)}{S_{2} \lambda_{3}}=0 .
$$

Finally, the estimable quantities are: $S_{1}, \lambda_{2}, p_{2}, \lambda_{3}\left(1-S_{2}\right), S_{2} p_{3}$.

For ring-recovery data, the constraints $\lambda_{j}=\lambda$ are known to make all parameters estimable (FrEEMAN and MorgAn, 1992). By applying the approach above, we 
checked that, when recoveries are mixed with recaptures, constraining the recovery rate to be constant makes $S_{2}$ and $p_{3}$ estimable. In addition, this constrained model is essentially full rank (as defined in Section 2) as shown by a procedure summarized in Appendix 2. We considered a fixed number of occasions for this example. However, following an approach similar to the one developed in Appendix 1 (see also CAtChPOle and Morgan, 1997), it can be easily shown that, for any $k \geq 3$, this model remains of full rank.

\section{Example 3: Markovian trap-dependence}

Heterogeneity of capture can result in a negative bias in estimates of survival (CARothers, 1973, 1979). Pradel (1993) shows how to handle an immediate trap-effect on capture - a particular kind of capture heterogeneity (SANDLAND and KIRKWOOD, 1981) - by considering a different capture probability depending upon whether an animal was caught on the previous occasion. Following Pradel's notation, we will denote $\left(\phi_{t}, p_{t} *_{m}\right)$ a model that allows trap-effects in different years.

By considering an unobservable state for non-capture at the previous occasion, these models may be rewritten in a straightforward way in a multistate framework. Indeed one can check easily that the likelihood of the model $\left(\phi_{t}, p_{t} *_{m}\right)$ is equal to the likelihood of a multistate model with:

$$
\Phi_{t}=\left[\begin{array}{ll}
\phi_{t} p_{t}^{*} & \phi_{t}\left(1-p_{t}^{*}\right) \\
\phi_{t} p_{t} & \phi_{t}\left(1-p_{t}\right)
\end{array}\right] ; \quad P_{t}=\left[\begin{array}{l}
1 \\
0
\end{array}\right]
$$

where $p_{t}^{*}$ and $p_{t}$ are the capture probabilities at time $t+1$ for an individual caught at the previous occasion $t$ or not, respectively. PRADEL (1993) points out critical parameter redundancy problems for this model. We are now able to investigate formally the parameter-redundancy in the presence of trap-dependence.

\section{Model $\left(\phi_{t}, p_{t} *_{m}\right)$ with interaction between time and trap-effect}

Assume $k=6$, for the $\operatorname{model}\left(\phi_{t}, p_{t} *_{m}\right)$, the matrix of probabilities is obtained through Step 1:

$\begin{array}{ccc}\phi_{1}\left(1-p_{2}^{*}\right) \phi_{2} p_{3} & 0 & \phi_{1}\left(1-p_{2}^{*}\right) \phi_{2}\left(1-p_{3}\right) \phi_{3} p_{4} \\ 0 & 0 & 0 \\ \phi_{2} p_{3}^{*} & 0 & \phi_{2}\left(1-p_{3}^{*}\right) \phi_{3} p_{4} \\ 0 & 0 & 0 \\ 0 & 0 & \phi_{3} p_{4}^{*} \\ 0 & 0 & 0 \\ 0 & 0 & 0 \\ 0 & 0 & 0 \\ 0 & 0 & 0 \\ 0 & 0 & 0\end{array}$

$$
\begin{array}{cc}
\phi_{1}\left(1-p_{2}^{*}\right) \phi_{2}\left(1-p_{3}\right) \phi_{3}\left(1-p_{4}\right) \phi_{4} p_{5} \\
0 \\
0 \\
0 \\
\phi_{2}\left(1-p_{3}^{*}\right) \phi_{3}\left(1-p_{4}\right) \phi_{4} p_{5} \\
0 \\
0 \\
\phi_{3}\left(1-p_{4}^{*}\right) \phi_{4} p_{5} \\
0 \\
0 \\
0 \\
\phi_{4} p_{5}^{*} \\
0 \\
0 \\
0
\end{array}
$$

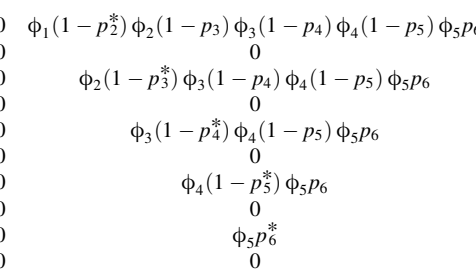

If the lines and columns corresponding to the non-observable state are deleted, one thus obtains exactly the same elementary probabilities as in the single state formulation of this model. Taking derivatives with respect to 
$\theta=\left(\phi_{1}, \phi_{2}, \phi_{3}, \phi_{4}, \phi_{5}, p_{2}^{*}, p_{3}, p_{3}^{*}, p_{4}, p_{4}^{*}, p_{5}, p_{5}^{*}, p_{6}, p_{6}^{*}\right)^{T}$, Step 2 leads to matrix $A$ with dimensions $(3 k-4) \times\left(\frac{k(k-1)}{2}\right)$ :

$$
A(\theta)=\left[\begin{array}{ccccc}
\frac{1}{\phi_{1}} & \frac{1}{\phi_{1}} & \cdots & 0 & 0 \\
0 & \frac{1}{\phi_{2}} & \cdots & 0 & 0 \\
\vdots & \vdots & \ddots & \vdots & \vdots \\
0 & 0 & \cdots & \frac{1}{p_{6}} & 0 \\
0 & 0 & \cdots & 0 & \frac{1}{p_{6}^{*}}
\end{array}\right] \text {, }
$$

with rank equal to 12 . The vector solution to (1) has no zero entries $(s=0)$, and so none of the original parameters is separately estimable. Indeed with Maple e.g., one can easily verify that in order to find the solution space of equation (1), a system of $d=2$ PDEs has to be solved, the solutions of which reduce to the

Table 2

The notations used in Imbert (1999)

\begin{tabular}{ll}
\hline$u_{i}=\phi_{i-1} p_{i}, i=3, \ldots, k$ & $\begin{array}{l}\text { Probability of surviving from occasion } i-1 \text { to occasion } i \text { and } \\
\text { being captured at occasion } i+1 \text { given that the individual has } \\
\text { not been captured at occasion } i \text { and has been released at occa- } \\
\text { sion } i-1 .\end{array}$ \\
\hline$u_{i}^{\prime}=\phi_{i-1} p_{i}^{\prime}, i=2, \ldots, k$ & $\begin{array}{l}\text { Probability of surviving from occasion } i-1 \text { to occasion } i \text { and } \\
\text { being captured at occasion } i+1 \text { given that the individual has } \\
\text { been captured at occasion } i \text { and has been released at occasion } \\
i-1 .\end{array}$ \\
\hline$v_{i}=\phi_{i-1}\left(1-p_{i}\right), i=3, \ldots, k$ & $\begin{array}{l}\text { Probability of surviving from occasion } i-1 \text { to occasion } i \text { and } \\
\text { not being captured at occasion } i+1 \text { given that the individual } \\
\text { has not been captured at occasion } i \text { and has been released at } \\
\text { occasion } i-1 .\end{array}$ \\
\hline$u_{i}^{\prime}=\phi_{i-1}\left(1-p_{i}^{\prime}\right), i=2, \ldots, k$ & $\begin{array}{l}\text { Probability of surviving from occasion } i-1 \text { to occasion } i \text { and } \\
\text { not being captured at occasion } i+1 \text { given that the individual } \\
\text { has been captured at occasion } i \text { and has been released at occa- } \\
\text { sion } i-1 .\end{array}$ \\
\hline$\delta_{i}=u_{i}+v_{i} u_{i+1}+v_{i} P v_{i+1} u_{i+2}+\ldots$ & $\begin{array}{l}\text { Probability of being seen again at occasion } i \text { or later given that } \\
\text { the individual has not been seen at occasion } i-1 .\end{array}$ \\
\hline$v_{i} v_{i+1} \ldots v_{k-1} u_{k}, i=3, \ldots, k$ & $\begin{array}{l}\text { Probability of being seen for the first time at occasion } i \text { given } \\
\text { that the individual will be seen later. }\end{array}$ \\
\hline$u_{i i}=\frac{u_{i}}{\delta_{i}}, i=3, \ldots, k$ &
\end{tabular}


$q=12$ functions proposed by Imbert (1999):

$$
\beta(\theta)=\left(u_{2}^{\prime}, u_{3}^{\prime}, u_{4}^{\prime}, u_{5}^{\prime}, u_{6}^{\prime}, \frac{v_{2}^{\prime} \delta_{3}}{1-u_{2}^{\prime}}, \frac{v_{3}^{\prime} \delta_{4}}{1-u_{3}^{\prime}}, \frac{v_{4}^{\prime} \delta_{5}}{1-u_{4}^{\prime}}, \frac{v_{5}^{\prime} \delta_{6}}{1-u_{5}^{\prime}}, d_{33}, d_{44}, d_{55}\right)^{T} .
$$

The notations used in IMBERT (1999) are synthesized in Table 2.

To make parameters estimable, PRADEL (1993) considered constrained models, including one with an additive effect of time and of last capture on the probability of recapture. In the following, we examine parameter redundancy for two forms of this additive model, both with $k=5$, with a logit (PRADEL, 1993) and a log link function (SANDLAND and KIRKWOOD, 1981), respectively.

\section{Additive time and trap-effects on a logit scale}

Step 1 is similar to the model $\left(\phi_{t}, p_{t} *_{m}\right)$, excepted that the elementary probabilities have to be expressed in the new parameterization:

$p_{t}^{*}=\frac{\exp \left(a_{t}+m\right)}{1+\exp \left(a_{t}+m\right)}, \quad t=2, \ldots, k \quad$ and $\quad p_{t}=\frac{\exp \left(a_{t}\right)}{1+\exp \left(a_{t}\right)}, \quad t=3, \ldots, k$

where $m$ (the trap-effect) and the $a_{t} \mathrm{~s}$ are parameters to be estimated. Step 2 requires the derivative matrix of $\Omega$ with respect to $\theta=\left(\phi_{1}, \phi_{2}, \phi_{3}, \phi_{4}, a_{2}\right.$, $\left.a_{3}, a_{4}, a_{5}, m\right)^{T}$ :

$A(\theta)=\left[\begin{array}{cccccccccc}\frac{1}{\phi_{1}} & \frac{1}{\phi_{1}} & \frac{1}{\phi_{1}} & \frac{1}{\phi_{1}} & 0 & 0 & 0 & 0 & 0 & 0 \\ 0 & \frac{1}{\phi_{2}} & \frac{1}{\phi_{2}} & \frac{1}{\phi_{2}} & \frac{1}{\phi_{2}} & \frac{1}{\phi_{2}} & \frac{1}{\phi_{2}} & 0 & 0 & 0 \\ 0 & 0 & \frac{1}{\phi_{3}} & \frac{1}{\phi_{3}} & 0 & \frac{1}{\phi_{3}} & \frac{1}{\phi_{3}} & \frac{1}{\phi_{3}} & \frac{1}{\phi_{3}} & 0 \\ 0 & 0 & 0 & \frac{1}{\phi_{4}} & 0 & 0 & \frac{1}{\phi_{4}} & 0 & \frac{1}{\phi_{4}} & \frac{1}{\phi_{4}} \\ h\left(a_{2}+m\right) & -h\left(-a_{2}-m\right) & -h\left(-a_{2}-m\right) & -h\left(-a_{2}-m\right) & 0 & 0 & 0 & 0 & 0 & 0 \\ 0 & h\left(a_{3}\right) & -h\left(-a_{3}\right) & -h\left(-a_{3}\right) & h\left(a_{3}+m\right) & -h\left(-a_{3}-m\right) & -h\left(-a_{3}-m\right) & 0 & 0 & 0 \\ 0 & 0 & h\left(a_{4}\right) & -h\left(-a_{4}\right) & 0 & h\left(a_{4}\right) & -h\left(-a_{4}\right) & h\left(a_{4}+m\right) & -h\left(-a_{4}-m\right) & 0 \\ 0 & 0 & 0 & h\left(a_{5}\right) & 0 & 0 & h\left(a_{5}\right) & 0 & h\left(a_{5}\right) & h\left(a_{5}+m\right) \\ h\left(a_{2}+m\right) & -h\left(-a_{2}-m\right) & -h\left(-a_{2}-m\right) & -h\left(-a_{2}-m\right) & h\left(a_{3}+m\right) & -h\left(-a_{3}-m\right) & -h\left(-a_{3}-m\right) & h\left(a_{4}+m\right) & -h\left(-a_{4}-m\right) & h\left(a_{5}+m\right)\end{array}\right]$

where $h\left(a_{2}\right)=\frac{1}{1+\exp \left(a_{2}\right)}$, etc. The rank of $A$ is 9 so that all the original parameters are separately estimable. Moreover, following both the simplification approach we advocate in Section 2 (see also Appendix 2) and using the relation

$$
\forall x \in \Re, h(x)+h(-x)=1
$$

one can check that this model is essentially full rank (see Appendix 2).

\section{Additive time and trap-effects on a log scale}

The new parameterization is:

$$
p_{t}^{*}=\exp \left(a_{t}+m\right), \quad t=2, \ldots, k \quad \text { and } \quad p_{t}=\exp \left(a_{t}\right), \quad t=3, \ldots, k,
$$


Step 2 leads to the derivative matrix:

$$
A(\theta)=\left[\begin{array}{cccccccccc}
\frac{1}{\phi_{1}} & \frac{1}{\phi_{1}} & \frac{1}{\phi_{1}} & \frac{1}{\phi_{1}} & 0 & 0 & 0 & 0 & 0 & 0 \\
0 & \frac{1}{\phi_{2}} & \frac{1}{\phi_{2}} & \frac{1}{\phi_{2}} & \frac{1}{\phi_{2}} & \frac{1}{\phi_{2}} & \frac{1}{\phi_{2}} & 0 & 0 & 0 \\
0 & 0 & \frac{1}{\phi_{3}} & \frac{1}{\phi_{3}} & 0 & \frac{1}{\phi_{3}} & \frac{1}{\phi_{3}} & \frac{1}{\phi_{3}} & \frac{1}{\phi_{3}} & 0 \\
0 & 0 & 0 & \frac{1}{\phi_{4}} & 0 & 0 & \frac{1}{\phi_{4}} & 0 & \frac{1}{\phi_{4}} & \frac{1}{\phi_{4}} \\
1 & g\left(a_{2}+m\right) & g\left(a_{2}+m\right) & g\left(a_{2}+m\right) & 0 & 0 & 0 & 0 & 0 & 0 \\
0 & 1 & g\left(a_{3}\right) & g\left(a_{3}\right) & 1 & g\left(a_{3}+m\right) & g\left(a_{3}+m\right) & 0 & 0 & 0 \\
0 & 0 & 1 & g\left(a_{4}\right) & 0 & 1 & g\left(a_{4}\right) & 1 & g\left(a_{4}+m\right) & 0 \\
0 & 0 & 0 & 1 & 0 & 0 & 1 & 0 & 1 & 1 \\
1 & g\left(a_{2}+m\right) & g\left(a_{2}+m\right) & g\left(a_{2}+m\right) & 1 & g\left(a_{3}+m\right) & g\left(a_{3}+m\right) & 1 & g\left(a_{4}+m\right) & 1
\end{array}\right]
$$

where $g\left(a_{3}\right)=\frac{1}{1-\exp \left(a_{3}\right)}$, etc. Following Step 3, the rank of $A$ is 8 , resulting in a rank deficiency $d=1$. Step 4 gives the solution to (1) as: $\alpha=\left(0,0,0,1,0,0,0,-\frac{1}{\phi_{4}}, 0\right)^{T}$.

By comparing with $\theta$, it comes that $\phi_{1}, \phi_{2}, \phi_{3}, a_{2}, a_{3}, a_{4}$ and $m$ are separately estimable (Step 5). Then, in Step 6, the following PDE has to be solved: $\frac{\partial f}{\partial \phi_{4}}-\frac{1}{\phi_{4}} \frac{\partial f}{\partial a_{5}}=0$ and solutions are $\beta=\left(\phi_{1}, \phi_{2}, \phi_{3}, a_{2}, a_{3}, a_{4}, m, a_{5}+\log \left(\phi_{4}\right)\right)^{T}$.

To sum up, if the logit-link function is considered, the model with additive time and trap-dependence is full rank, while the log-link results in a rank deficiency. VIALLEFONT (1995) has noticed such a phenomenon for the CJS model with two groups. She demonstrated that this model was full rank if and only if the link function was not the logarithm or any linear function of the logarithm.

One can prove by induction (as for Example 1 and 2) that, for any greater $k$, parameter-redundancy for models $\left(\phi_{t}, p_{t} *_{m}\right),\left(\phi_{t}, p_{\log (t+m)}\right)$ and $\left(\phi_{t}, p_{\log i t(t+m)}\right)$ is similar to that found here (e.g. IMBERT, 1999 for the last model).

\section{Discussion}

Our results confirm that the CMF approach is quite efficient to check formally for parameter redundancy in product-multinomial models. With the Arnason-Schwarz model, a straightforward generalization of the well known CJS model to several states, the estimable parameters are analogous to those of the single state case. In what follows, we discuss in sequence:

- The application of our approach to more complex models;

- The interest of automatic determination of the estimable parameters; 
- The interest of extension theorems;

- The usefulness of our approach when a model is applied to a particular dataset.

Little adjustments are needed to make the same approach valid for more complex capture-recapture models. Instances of such cases concern models for several groups (e.g. male and female) (CATCHPOLE et al., 2002) and age-dependent multistate models (CATCHPOLE et al., 1995). Indeed, all such models remain in the wide class of product-multinomial models to which all our results apply. More general models for mixtures of information with non-observable states can be treated in the same way. In this framework, models with immediate trap-dependence or to handle temporary emigration (KENDALL and NichOLS, 2002) can be treated as multistate models and then parameter redundancy can be checked. The link function is then critical in determining the number of estimable parameters.

Obviously, in some cases such as the CJS model, estimable parameters that are solutions to partial differential equations could be found by visual inspection of the matrix of recapture probabilities. However this ad hoc method quickly becomes impractical, especially for multistate models. On the contrary, the use of symbolic calculus software makes it possible to solve system of PDEs in a rigorous way and thus provides, in an automatic and reliable fashion, functions of the original parameters which are estimable. The reliability of this approach, based upon symbolic calculations, appears quite good for all the cases treated even if sampling occasions are added or data are missing.

On the one hand, although the $\mathrm{CMF}$ approach as generalized here applies to a problem of fixed size, extension results (Appendix 1) make it easy to obtain general results on parameter redundancy independent of the number of occasions.

On the other hand, missing data can result in making a full rank model parameter redundant (CATCHPOLE and MoRgan, 2001). The present approach still holds provided some adjustments are applied in the matrix of probabilities (CATCHPOLE et al., 2002): the probabilities corresponding to the missing data in the $m$-array have to be removed and the last probabilities for individuals never seen again initially skipped (see Section 3) have to be taken into account to obtain multinomials. This procedure has been applied (Gimenez and Choquet, unpublished results) to an example using a multistate study recently published (SCOFIELD et al., 2001). We provide the corresponding Maple code needed to check for parameter redundancy in presence of missing data at URL: ftp://ftp.cefe.cnrs-mop.fr/biom/ PRM.

Besides, there may exist full rank models that can have for some data sets i.e. for certain values of the vector of parameters, a rank deficiency in the matrix of derivatives resulting in a likelihood surfaces with a flat ridge, i.e. non-unique MLEs (CATChPOLE and MoRgan, 1997). In this paper, we give a simple manner to distinguish between these conditionally full rank models and essentially full rank models nevertheless the method gives only a sufficient condition that a model is essentially full rank. 
In the near future, we hope to be able to implement the approach presented here in software for fitting capture-recapture models. This would allow practitioners to handle parameter redundancy in multistate models in an automatic way.

Appendix 1: Extension result for the Arnason-Schwarz model

Consider the Arnason-Schwarz model with $k=3$ capture occasions and $a=2$ states where we have the vector of parameters $\theta=\left(\phi_{1}^{11}, \phi_{1}^{21}, \phi_{1}^{12}, \phi_{1}^{22}, p_{2}^{1}, p_{2}^{2}\right.$, $\left.\phi_{2}^{11}, \phi_{2}^{21}, \phi_{2}^{12}, \phi_{2}^{22}, p_{3}^{1}, p_{3}^{2}\right)^{T}$. If we work through the nonzero elements of the matrix of probabilities $\Omega$ (see (3)), the row rank of the standardized derivative matrix (CATChPole and Morgan, 2001) is 10 based on symbolic computation with Maple. Moreover there are two linearly independent solutions to equation (1),

$\alpha_{1}=(0,0,0,0,0,0,-1,-1,0,0,1,0)^{T}$ and $\alpha_{2}=(0,0,0,0,0,0,0,0,-1,-1,0,1)^{T}$.

Thus for $k=3$ and $a=2$ the Arnason-Schwarz model has deficiency 2. There are 6 separately estimable parameters $\phi_{1}^{11}, \phi_{1}^{21}, \phi_{1}^{12}, \phi_{1}^{22}, p_{2}^{1}$ and $p_{2}^{2}$ corresponding to the same positions of the zeros in $\alpha_{1}$ and $\alpha_{2}$ and 2 other estimable combinations of parameters $\phi_{2}^{11}, \phi_{2}^{12}, \phi_{2}^{21}, \phi_{2}^{22}, p_{3}^{1}$ and $p_{3}^{2}$. We now prove by induction that, for any study with $k \geq 3$ capture occasions, the two only nonzero solutions to (1) are

$\alpha_{1}^{k}=(\overbrace{0,0, \ldots, 0}^{6(k-2)},-1,-1,0,0,1,0)^{T}$ and $\alpha_{2}^{k}=(\overbrace{0,0, \ldots, 0}^{6(k-2)}, 0,0,-1,-1,0,1)^{T}$.

When the study is extended by one year, the probability matrix $\Omega$ gains one column of block matrices $W$ with $W_{i}=\prod_{j=i}^{k} \Phi_{j} D\left(P_{j+1}\right), 1 \leq i \leq k$. Following САтсHPOLE and MORGAN (2001, p. 595), we partition $\theta$ in $\left(\theta^{(1)}, \theta^{(2)}\right)$ with

$$
\begin{aligned}
& \theta^{(1)}=\left(\phi_{1}^{11}, \phi_{1}^{21}, \phi_{1}^{12}, \phi_{1}^{22}, p_{2}^{1}, p_{2}^{2}, \ldots, \phi_{k-1}^{11}, \phi_{k-1}^{12}, \phi_{k-1}^{21}, \phi_{k-1}^{22}, p_{k}^{1}, p_{k}^{2}\right)^{T}, \\
& \theta^{(2)}=\left(\phi_{k}^{11}, \phi_{k}^{12}, \phi_{k}^{21}, \phi_{k}^{22}, p_{k+1}^{1}, p_{k+1}^{2}\right)^{T}
\end{aligned}
$$

and $\alpha^{k+1}=(\alpha, \beta)$ in a similar way. Then (1) becomes

$$
\alpha^{k+1^{T}} \Delta^{k+1}=\left[\alpha^{T}, \beta^{T}\right]\left[\begin{array}{cc}
\Delta^{k} & X \\
0 & B
\end{array}\right]=\left[\alpha^{T} \Delta^{k}, \alpha^{T} X+\beta^{T} B\right]
$$

The matrix $B$ is obtained by differentiating vec $(W)$ with respect to the components of $\theta^{(2)}$ and by using Maple, $B$ has row rank equal to 4 . Therefore, it can be easily seen that the space spanned by the rows of $B$ is the row space of the matrix $C=\left[\begin{array}{cccccc}\ldots & 1 & 0 & \ldots & 0 & 0 \\ \ldots & 0 & 1 & \ldots & 0 & 0 \\ \ldots & 0 & 0 & \ldots & 1 & 0 \\ \ldots & 0 & 0 & \ldots & 0 & 1\end{array}\right]$ where the points stand for non-useful components 
for our purpose. Then, in order that $\alpha^{k+1^{T}} \Delta^{k+1}=0$, (A1) requires that $\alpha^{T} \Delta^{k}=0$, which by the induction hypothesis implies that $\alpha=\alpha_{1}^{k}$ or $\alpha=\alpha_{2}^{k}$ or $\alpha=(0, \ldots, 0)^{T}$ with dimensions $6(k-1) \times 1$. But if $\alpha=\alpha_{1}^{k}$ or $\alpha=\alpha_{2}^{k}$, one can easily check that $\alpha^{T} X$ is equal to a row vector of the form $(\ldots, 0,0, \ldots, 0,0)$ or a different vector of the same form $(0,0, \ldots, 0,0, \ldots)$ which both clearly do not belong to the row space of $C$, and consequently $\left[\alpha^{T} \Delta_{k}, \alpha^{T} X+\beta^{T} B\right]$ has no solution. Hence, the only two solutions to (A1) are $\alpha=(0, \ldots, 0)^{T}$ and $\beta=(-1,-1,0,0,1,0)^{T}$ or $\beta=(0,0,-1,-1,0,1)^{T}$.

In the preceding demonstration, the number of states was assumed to be 2 . However it may be proved little by little that for any study with $k \geq 3$ capture occasions and $a \geq 2$ states, the rank deficiency is $a$ and the $a$ solutions to (1) are:

$$
\begin{aligned}
& \alpha_{1}^{k}=(\overbrace{0, \ldots, 0}^{(k-2) a(a+1)}, \underbrace{-1, \ldots,-1}_{a}, \overbrace{0, \ldots, 0}^{a(a-1)}, \underbrace{1,0, \ldots, 0}_{a})^{T}, \\
& \alpha_{2}^{k}=(\overbrace{0, \ldots, 0}^{(k-2) a(a+1)}, \underbrace{0, \ldots, 0}_{a}, \overbrace{-1, \ldots,-1}^{a}, \underbrace{0, \ldots, 0}_{a(a-2)}, \underbrace{0,1, \ldots, 0}_{a})^{T}, \ldots, \\
& \alpha_{a}^{k}=(\overbrace{0, \ldots, 0}^{(k-2) a(a+1)}, \underbrace{0, \ldots, 0}_{a(a-1)}, \overbrace{-1, \ldots,-1}^{a}, \underbrace{0,0, \ldots, 1}_{a})^{T} .
\end{aligned}
$$

Hence, there are $(k-2) a(a+1)$ separately estimable parameters and $a^{2}$ estimable elements of $\Phi_{k} D\left(P_{k+1}\right)$.

Appendix 2: Distinguishing between essentially and conditionally full rank models

2a: The model for mixtures of information is shown to be essentially of full rank. The proof consists in four steps of simplification of the matrix $A(\theta)$ up to an integer matrix $L(\theta)$ of full rank. Each step labelled $i$ is equivalent to a pre-multiplication of $A(\theta)$ by a non singular matrix $D^{(i)}(\theta)$.

\section{Step 1:}

$$
D^{(1)}=\left(\begin{array}{ccccc}
S_{1} \bar{S}_{1} & 0 & 0 & 0 & 0 \\
0 & S_{2} \bar{S}_{2} & 0 & 0 & 0 \\
0 & 0 & \lambda & 0 & 0 \\
0 & 0 & 0 & p_{2} \bar{p}_{2} & 0 \\
0 & 0 & 0 & 0 & p_{3}
\end{array}\right) \Rightarrow L^{(1)}=D^{(1)} A(\theta)=\left(\begin{array}{cccccc}
\bar{S}_{1} & -S_{1} & \bar{S}_{1} & \bar{S}_{1} & 0 & 0 \\
0 & 0 & \bar{S}_{2} & -S_{2} & \bar{S}_{2} & -S_{2} \\
0 & 1 & 0 & 1 & 0 & 1 \\
\bar{p}_{2} & 0 & -p_{2} & -p_{2} & 0 & 0 \\
0 & 0 & 1 & 0 & 1 & 0
\end{array}\right)
$$




\section{Step 2:}

$D^{(2)}=\left(\begin{array}{ccccc}1 & 0 & 0 & 0 & 0 \\ 0 & 1 / S_{2} & 1 & 0 & -\bar{S}_{2} / S_{2} \\ 0 & 0 & 1 & 0 & 0 \\ 0 & 0 & 0 & 1 & 0 \\ 0 & 0 & 0 & 0 & 1\end{array}\right) \Rightarrow L^{(2)}=D^{(2)} L^{(1)}=\left(\begin{array}{cccccc}\bar{S}_{1} & -S_{1} & \bar{S}_{1} & \bar{S}_{1} & 0 & 0 \\ 0 & 1 & 0 & 0 & 0 & 0 \\ 0 & 1 & 0 & 1 & 0 & 1 \\ \bar{p}_{2} & 0 & -p_{2} & -p_{2} & 0 & 0 \\ 0 & 0 & 1 & 0 & 1 & 0\end{array}\right)$

Step 3:

$D^{(3)}=\left(\begin{array}{ccccc}1 / \bar{S}_{1} & S_{1} / \bar{S}_{1} & 0 & 0 & 0 \\ 0 & 1 & 0 & 0 & 0 \\ 0 & -1 & 1 & 0 & 0 \\ 0 & 0 & 0 & 1 & 0 \\ 0 & 0 & 0 & 0 & 1\end{array}\right) \Rightarrow L^{(3)}=D^{(3)} L^{(2)}=\left(\begin{array}{cccccc}1 & 0 & 1 & 1 & 0 & 0 \\ 0 & 1 & 0 & 0 & 0 & 0 \\ 0 & 0 & 0 & 1 & 0 & 1 \\ \bar{p}_{2} & 0 & -p_{2} & -p_{2} & 0 & 0 \\ 0 & 0 & 1 & 0 & 1 & 0\end{array}\right)$

Step 4:

$D^{(4)}=\left(\begin{array}{ccccc}1 & 0 & 0 & 0 & 0 \\ 0 & 1 & 0 & 0 & 0 \\ 0 & 0 & 1 & 0 & 0 \\ p_{2} & 0 & 0 & 1 & 0 \\ 0 & 0 & 0 & 0 & 1\end{array}\right) \Rightarrow L=D^{(4)} L^{(3)}=\left(\begin{array}{cccccc}1 & 0 & 1 & 1 & 0 & 0 \\ 0 & 1 & 0 & 0 & 0 & 0 \\ 0 & 1 & 0 & 1 & 0 & 1 \\ 1 & 0 & 0 & 0 & 0 & 0 \\ 0 & 0 & 1 & 0 & 1 & 0\end{array}\right)$

2b: Concerning the trap dependence model, the integer matrix $L(\theta)$ of full rank is displayed without the several steps of pre-multiplication of $A(\theta)$ by non-singular matrices.

$$
L=\left(\begin{array}{llllllllll}
0 & 1 & 1 & 1 & 0 & 0 & 0 & 0 & 0 & 0 \\
0 & 0 & 0 & 0 & 1 & 1 & 1 & 0 & 0 & 0 \\
0 & 0 & 0 & 0 & 0 & 0 & 0 & 1 & 1 & 0 \\
0 & 0 & 0 & 0 & 0 & 0 & 0 & 0 & 0 & 1 \\
1 & 0 & 0 & 0 & 0 & 0 & 0 & 0 & 0 & 0 \\
0 & 1 & 0 & 0 & 1 & 0 & 0 & 0 & 0 & 0 \\
0 & 0 & 1 & 0 & 0 & 1 & 0 & 1 & 0 & 0 \\
0 & 0 & 0 & 1 & 0 & 0 & 1 & 0 & 1 & 0 \\
0 & 0 & 0 & 0 & 1 & 0 & 0 & 1 & 0 & 0
\end{array}\right)
$$

Acknowledgements

The authors thank Edgardo S. Cheb-Terrab for helping us in the use of the Maple PDEtools package and Anne Viallefont, Michael Schaub and three anonymous referees for very helpful comments. 


\section{References}

Arnason, A. N., 1973: The estimation of population size, migration rates and survival in a stratified population. Research in Population Ecology 15, 1-8.

Brownie, C., Hines, J. E., Nichols, J. D., Pollock, K. H., and Hestbeck J. B., 1993: Capture recapture studies for multiple strata including non-Markovian transitions. Biometrics 49, 1173-1187.

BurnhaM, K. P., 1993: A theory for combined analysis of ring recovery and recapture data. Marked individual in the study of bird population. In J.-D. Lebreton and P. M. North (eds.): Marked Individuals in the Study of Bird Population. Birkhauser Verlag, Basel, Switzerland, 199-213.

Burnham, K. P. and Anderson, D. R., 1998: Model selection and inference, a practical Information theoretic approach. Springer, New-York.

CArothers, A. D., 1973: The effects of unequal catchability on Jolly-Seber estimates. Biometrics 29, 79-100.

CARothers, A. D., 1979: Quantifying unequal catchability and its effect on survival estimates in an actual population. Journal of Animal Ecology 48, 863-869.

Catchpole, E. A., Freeman, S. N., and Morgan, B. J. T., 1995: Modelling age variation in survival and reporting rates for recovery models. Journal of Applied Statistics 22, 597-609.

Catchpole, E. A., Freeman, S. N., and Morgan, B. J. T., 1996: Steps to parameter redundancy in age-dependent recovery models. Journal of the Royal Statistical Society Series B 58, 763-774.

Catchpole, E. A. and Morgan, B. J. T., 1997: Detecting parameter redundancy. Biometrika 84, 187-196.

Catchpole, E. A., Morgan, B. J. T., and Freeman, S. N., 1998: Estimation in parameter redundant models. Biometrika 85, 462-468.

Catchpole, E. A. and Morgan, B. J. T., 2001: Deficiency of parameter redundant models. Biometrika 88, 593-598.

Catchpole, E. A., Morgan, B. J. T., and Viallefont, A., 2002: Solving problems in parameter redundancy using computer algebra: Journal of Applied Statistics 29, 625-636.

Cheb-Terrab, E. S. and von Bulow, K., 1995: A Computational Approach for the Analytical Solving of Partial Differential Equations. Computer Physics Communications 90, 102-116.

Choquet, R. Reboulet, A. M., Pradel, R. Gimenez, O., and Lebreton, J.-D., 2003: User's manual for M-SURGE 1.0. CEFE/CNRS, Mimeographed document.

Cormack, R. M., 1964: Estimates of survival from the sighting of marked animals. Biometrika 51, 429-438.

Frederiksen, M. and Bregnballe, T., 2000: Factors affecting survival and colony fidelity of adult Cormorants: a combined analysis of recoveries and resightings. Journal of Animal Ecology 69, 737-752.

Freeman, S. N. and Morgan, B. J. T., 1992: A modelling strategy for recovery data from birds ringed as nestlings. Biometrics 48, 217-235.

Golub, G. H. and van LoAn, C. F., 1996: Matrix Computations, 3rd Edition, Johns Hopkins University Press, Baltimore.

Graybill, F. A., 1983: Matrices with Applications in Statistics, 2nd Edition, Belmont, Wadsworth.

Hestbeck, J. B., Nichols, J. D., and Malecki, R. A., 1991: Estimates of movement and site fidelity using mark-resight data of wintering Canada Geese. Ecology 72, 523-533.

IMBERT, O., 1999: Problèmes d'identifiabilité dans les modèles de capture-recapture avec taux de capture hétérogène. Unpublished D.E.A. report. University of Montpellier II.

Jolly, G. M., 1965: Explicit estimates from capture-recapture data with both death and immigrationstochastic models. Biometrika 52, 225-247.

Kendall, W. L. and NichOls, J. D., 2002: Estimating state-transition probabilities for unobservable states using capture-recapture/resighting data. Ecology, 83, 3276-3284.

Lebreton, J.-D., 2001: The use of bird rings in the study of survival. Ardea, 89, 85-100.

Lebreton, J.-D., Burnham, K. P., Clobert, J., and Anderson, D. R., 1992: Modeling survival and testing biological hypotheses using marked animals: a unified approach with case studies. Ecological Monographs 62, 67-118.

2031633 Biom. Journal (7/2003)

Art.: 0580/Gimenez

Bearb.: Sch.

SIGNA 18.7.2003 ges.Seiten $19 \quad\{$ p_1\}bio/bio03_07/0580/bio0580u.3d

Literaturstellen: 26 <bibitem> nur für Literatur verwenden, keine Leerzeilen damit formatieren, die exakte Anzahl der LitStellen wird für SGML benötigt 
Lebreton, J.-D., Morgan, B. J. T., Pradel, R., and Freeman, S. N., 1995: A simultaneous survival rate analysis of dead recovery and live recapture data. Biometrics 51, 1418-1428.

Lebreton, J.-D., Almeras, T., and Pradel, R., 1999: Competing events, mixture of information and multistratum recapture models. Bird Study 46, S39-46.

Lebreton, J.-D. and Pradel, R., 2002: Multistate recapture models: modelling incomplete individual histories. Journal of Applied Statistics, 29: 353-369.

Lebreton, J.-D., Hines, J. E., Pradel, R., Nichols, J. D., and Spendelow, J. A., 2003: Estimation by capture-recapture of recruitment and dispersal over several sites. Oikos 101, 253-264.

McCullagh, P. and Nelder, J., 1989: Generalized Linear Models, 2nd Edition. London, Chapman and Hill.

Nichols, J. D., Hines, J. E., Pollock, K. H., Hinz, R. L., and LinK, W. A., 1994: Estimating breeding proportions and testing hypotheses about costs of reproduction with capture-recapture data. Ecology 75, 2052-2065.

Nichols, J. D. and Kendall, W. L., 1995: The use of multistate capture-recapture models to address questions in evolutionary ecology. Journal of Applied Statistics 22, 835-846.

Pradel, R., 1993: Flexibility in Survival Analysis from Recapture Data: Handling Trap-Dependence. In J.-D. LeBreton and P. M. North (eds.): Marked Individuals in the Study of Bird Population. Birkhäuser Verlag, Basel, Switzerland, 29-37.

PRADEL, R. and Lebreton J.-D., 1999 : Comparison of different approaches to the study of local recruitment of breeders. Bird Study 46 (suppl.), S. 74-81.

SAndland, R. L. and Kirkwood, G. P., 1981: Estimation of survival in marked populations with possibly dependent sighting probabilities. Biometrika 68, 531-541.

Schwarz, C. J., Schweigert J. F., and Arnason, A. N., 1993: Estimating migration rates using tagrecovery data. Biometrics $\mathbf{4 9}, 177-193$.

SCOFIEld, R. P., Fletcher D. J., and Robertson, R., 2001: Titi (Sooty Shearwaters) on Whero Island: Analysis of Historic Data Using Modern Techniques. Journal of Agricultural, Biological, and Environmental Statistics 6: $268-280$.

Seber, G. A. F., 1965: A note on the multiple recapture census. Biometrika 52, 249-259.

VialleFOnT, A., 1995: Robustesse et flexibilité des analyses démographiques par capture-recapture. De l'estimation de la survie à la détection de compromis évolutifs. Unpublished $\mathrm{PhD}$ thesis. University of Montpellier II.

Viallefont, A., Lebreton, J.-D., Reboulet, A.-M., and Gory, G., 1998: Parameter identifiability and model selection in capture-recapture models : a numerical approach. Biometrical Journal 40, 1-13.

White, G. C. and Burnham K. P., 1999: Program MARK: survival estimation from populations of marked animals. Bird Study 46, 120-139. 